\title{
Progress in Understanding the Role of Carotid Endarterectomy
}

The North American Symptomatic Carotid Endarterectomy Trial (NASCET) was initiated because of genuine uncertainty about the appropriateness of the increasing popularity of this procedure. Evidence of this uncertainty was the geographic disparity in its application. In the United States it was performed 27 and 8 times as often on a population based calculation as in the United Kingdom and Canada respectively.' As well, the numbers performed annually in the United States fluctuated widely. In 1980 approximately 70,000 were performed and the numbers for 1985 and 1988 were 107,000 and 68,000 respectively. ${ }^{2} \mathrm{~A}$ study initiated by the American Medicare to define the application of endarterectomy, agreed upon by a panel of experts in the field, tested its use against an "appropriateness index". It concluded that one-third of those who received the procedure were appropriate, one-third were not appropriate and one-third were of uncertain appropriateness. ${ }^{3}$ All of this uncertainty existed despite the fact that a voluminous literature describing scores of thcusands of case reports had accumulated, two negative randomized trials of small size had been conducted and one million patients had been submitted to the procedure between 1954 when it was introduced and 1985.4.5

It is well known to the readers of this Journal that the NASCET discontinued entry of patients with severe stenosis (greater or equal to $70 \%$ by strict arteriographic linear-diameter measurement) after 659 patients had been randomized to receive best medical care alone or best medical care plus endarterectomy. ${ }^{6}$ The positive benefit of surgery was corroborated by a parallel trial (European Carotid Surgery Trial, ECST) conducted in Europe and reported in the same year. ${ }^{7} \mathrm{~A}$ trend in this direction was identified in a small American Veterans Administration trial which stopped because of the clear benefit indicated in the North American and European trials. ${ }^{8}$

Two unpredicted facts leap from the reported results of the initial part of this trial. The first is the extraordinary power of a randomized clinical trial to provide an answer that was elusive despite 35 years of accumulated anecdotes. 659 patients gave a final answer denied by anecdotes from one million. Secondly, patients with symptoms related to severe stenosis of the internal carotid artery stenosis proved to have a higher risk of ischemic stroke than had been previously known. Less visible in the report was the importance of the Canadian contribution to this study. Although less than one-quarter of the centers involved in the trial were Canadian, they contributed half of the patients to this first phase of NASCET.

Neither NASCET nor ECST were able to report benefit or harm for patients who had less than $70 \%$ stenosis. By NASCET standards the European trial admitted a substantial number of patients to their "severe" group who would not be severe by the NASCET measurement. When the European arteriograms were remeasured by NASCET standards there was no longer a disparity favoring NASCET's beneficial results over those of the European trial. The stroke-free survival curves for these similar patients are alike. ${ }^{9}$
Both of these trials continue to randomize patients who have moderate stenosis associated with appropriate symptoms. There are no data by which we can state with firm conviction that these patients should or should not be submitted to endarterectomy and it is imperative that these two ongoing trials continue to seek answers to this question. By March 31, 1994 the European trial will stop recruitment with 2,000 patients with moderate disease. To achieve its objective of 2,100 moderate patients, the North American trial needs to randomize 500 more patients. This can be achieved with full cooperation from the present centers by late 1995 or early 1996. An extra burst of activity could reduce the time until we have the final answers by several months.

Those of us conducting NASCET are watching with interest the impact of the early results on: the acceptance of the results and their interpretation, the overall use of endarterectomy, the referral of appropriate patients for endarterectomy, the referral of appropriate patients to best medical care and the referral of patients with stenosis due to moderate disease for evaluation in the ongoing phase of NASCET. Evidence has been presented by Dyken that for the ten months of 1991 after the initial Clinical Alert signalled our findings for patients with severe disease there was a $20-25 \%$ increase in the total number of endarterectomies carried out under Medicare in the United States compared to the decline which had been witnessed in the previous four years. ${ }^{2}$ The paper in this issue from one of our centers provides an interesting indication of what might be happening in many communities. ${ }^{10}$

Neurologists, neurovascular surgeons and vascular surgeons must now shape the pattern of practice by ensuring that patients who have symptoms and lesions comparable to those in NASCET receive this procedure in the best and safest way. They and their patients must also be aware that there are situations in which we remain ignorant of benefit of this operation. They must ask their patient's help in determining the answers and must ask their practicing colleagues to be patient while we seek the answers. We need the help of practitioners and patients in the ongoing NASCET trial for those symptomatic with less than $70 \%$ stenosis by arteriographic linear diameter measurement. We need their patience while we await the results of the two major ongoing trials to determine whether or not there is a group of asymptomatic patients who will benefit by the removal of the asymptomatic stenosed segment of the carotid artery. "To every patient in these asymptomatic categories, including those with bilateral disease, disease which remains asymptomatic despite ultrasound evidence of progression of the stenosing lesion, or who are about to undergo coronary bypass surgery in the presence of asymptomatic carotid lesion(s), we must tell them candidly and honestly that there is, as yet, no scientific data from scrupulous trials, which affirms that they will benefit. We want to know, so do they. We and they can help to get the answers! 
Without the use of reasonable caution, new treatments and new light cast on old treatments can be extrapolated beyond what has been disclosed by scientific study. A recent report of "What's New in Surgery" featured in Bulletin of the prestigious American College of Surgeons contained the astounding and totally erroneous statement that: "In summary, these randomized clinical trials, (referring to NASCET and ECST) all show that all symptoms that can be attributed to carotid artery disease should be operated upon". ${ }^{12}$ A lone exception was made: "Asymptomatic stenoses less than $70 \%$ are still being evaluated". Equally misleading and misinterpreting of what is known from scientific evaluation is a statement in a recent Editorial. ${ }^{13}$ "It is our opinion that lesions that reach a threshold of $50 \%$ or greater stenosis (75\% area-reducing stenosis), regardless of the method of measurement, should prompt consideration for intervention." Authors and editors must help to maintain the integrity of what we know and not counsel the extension of trial results to embrace the unknown or even the uncertain. Fortunately data from clinical trials are becoming available to eliminate the need to proceed on the basis of experiential and anecdotal judgement. At the same time opinions persist which believe that we can depend on non-randomized historical controls and uncontrolled case-studies to find answers without the expenditure of time, energy and money for randomized trials. ${ }^{14}$ Would that it were so!

While the process of evaluating endarterectomy continues we must determine the best way to investigate patients suspected of having symptoms related to a carotid stenosing lesion. Despite the disappointing correlation of ultrasound to angiography, as reported by NASCET, the VA trial, the current paper by Coyne and Wallace ${ }^{10}$ (in this issue), as well as a number of papers from other major vascular centers, ultrasound stands as the best screening tool for the patients with symptomatic lesions who might appropriately be offered endarterectomy. ${ }^{15.16 .17}$ Patients with focal symptoms and signs in the hemisphere or retina are those who should be asked to undergo investigation.

When the symptomatic patient has an ultrasound it is the writers' opinion that if the lesion looks "severe" this is insufficient investigation to be followed by endarterectomy. The ultrasound generally practiced today is not able yet to identify with sufficient precision the exact degree of stenosis. Contrary opinions have been expressed but they are without controls or correlation to what is known from clinical trials. ${ }^{18}$ The cut-off point at $70 \%$ by NASCET arteriographic methods has created the watershed: below $70 \%$ we have no scientific basis to recommend endarterectomy; above it we are compelled to consider it.

Ultrasound fails to identify a number of near-occlusions, important candidates for endarterectomy. Ultrasound fails to identify predictably the hazardous intraluminal thrombus which occurs not uncommonly in tight stenosis. The best evidence to date is that these individuals should be submitted to a month on anticoagulant therapy and then considered for endarterectomy. ${ }^{19}$ Ultrasound does not detect ulceration, an important indicator of a worsened prognosis for these patients. ${ }^{20}$ Finally, ultrasound cannot visualize satisfactorily, the intracranial circulation, including major intracranial artery stenosis or occlusion. Intracranial carotid stenosis adds substantially to the gravity of the outlook facing these patients. ${ }^{21}$ Transcranial Doppler and Magnetic Resonance Angiography (MRA) are not yet a reliable substitute for conventional arteriography in this regard. MRA is improving in its abilities to measure intracranial and extracranial stenosis but it tends to over-read the intruding lesions, and still has a problem with signal-void. Intracranial aneurysms were detected in the intracranial circulation of $2.8 \%$ of NASCET's patients. Four of them were of a size to be judged in need of surgery. Two of them ruptured before this was done, one fatally. The overlap of age-groups in which aneurysms and carotid stenosis occur requires that pre-endarterectomy imaging studies seek fusiform carotid or berry aneurysms. In the near future this will be a predictable MRA capability.

Patients with screening ultrasound studies which identify moderate stenosis in the presence of appropriate ipsilateral symptoms should be considered as candidates for confirmatory arteriography. If the initial films identify no lesion between 30 and $70 \%$, no further views need be taken. If there is a lesion in this range, selective intracranial views should be obtained and the patient referred to a NASCET center.

It has not been established that endarterectomy benefits patients without symptoms, or with vague complaints called "non-hemisphere" symptoms by some (dizziness, light-headedness, forgetfulness, memory lapses, mild unsteadiness, etc.). Therefore it is quite uncertain that there is reason to submit patients with only these non-carotid territory symptoms, even in the presence of a cervical bruit, to investigation with ultrasound.

It is comforting to those who deal with stroke and strokethreatened patients to know that within five years it is likely that all indications and all lack of indications for carotid endarterectomy will be validated by clinical trials. In the meantime we urge the readers of this Journal to be frank with their patients when they are recommending endarterectomy in situations where weak or no data exist. We urge them as well, if they have symptomatic patients with moderate stenosis to call the NASCET hot-line at 1-800-565-6331 to learn of the nearest center participating in this trial.

\section{H.J.M. Barnett and Heather Meldrum London, Ontario}

\section{REFERENCES}

1. Warlow C, Whisnant JP, Dyken ML: Carotid endarterectomy. In: Cerebrovascular Diseases, eds. F. Plum and W. Pulsinelli. Raven Press, New York. pp 43-48, 1985.

2. Dyken ML: Controversies in Stroke: Past and Present. The Willis Lecture. Stroke 1993; 24: 1251-1258.

3. Winslow Cm, Solomon DH, Chassin MR, Kosecoff J, Merrick NJ, Brook RH: The appropriateness of carotid endarterectomy. N Engl J Med 1988; 318: 721-727.

4. Fields WS, Maslenikov V, Meyer JS, Hass WK, Remington RD, Macdonald M: Joint study of extracranial arterial occlusion. V. Progress report of prognosis following surgery or nonsurgical treatment for transient cerebral ischemic attacks and cervical carotid artery lesions. JAMA 1970; 211: 1993-2003.

5. Shaw DA, Venables GS, Cartlidge NEF, Bates D, Dickinson PH: Carotid endarterectomy in patients with transient cerebral ischemia. J Neurol Sci 1984; 64: 45-53.

6. North American Symptomatic Carotid Endarterectomy Trial Collaborators: Beneficial effect of carotid endarterectomy in symptomatic patients with high-grade carotid stenosis. New Engl J Med 1991; 325: 445-453.

7. European Carotid Surgery Trialists' Collaborative Group. MRC European Carotid Surgery Trial: Interim results for symptomatic patients with severe $(70-99 \%)$ or with mild $(0-29 \%)$ carotid stenosis. Lancet 1991; 337: 1235-1243. 
8. Mayberg MR, Wilson SE, Yatsu F, Weiss DG, Messina L, Hershey LA, Colling C, Eskridge J, Deykin D, Winn R: Carotid endarterectomy and prevention of cerebral ischemia in symptomatic carotid stenosis. JAMA 1991; 266: 3289-3294.

9. Barnett HJM, Warlow CP: Carotid endarterectomy and the measurement of stenosis. Stroke 1993; 24: 1281-1284.

10. Coyne TJ, Wallace MC: Surgical referral for presumed carotid artery stenosis - the influence of the North American Symptomatic Carotid Endarterectomy Trial and the role of carotid angiography. Can J Neuro Sci 1994; 21: 129-132.

11. The Asymptomatic Carotid Atherosclerosis Study Group: Study design for randomized prospective trial of carotid endarterectomy for asymptomatic atherosclerosis. Stroke 1989; 20: 844-849.

12. Johnson G: What's new in vascular surgery. ACS Bulletin 1994; 79: 85-88.

13. Hobson RW and Strandness E: Carotid artery stenosis: What's in the measurement? J Vasc Surg 1993; 1069-1070.

14. Bergan JJ: Carotid surgery trials teach the obvious - again. Heart Disease and Stroke 1993: 269-270.

15. Howard G, Chambless LE, Baker WH, et al: A multicenter validation study of Doppler ultrasound versus angiography. J Stroke Cerebrovasc Dis 1991; 1: 166-173.
16. Riles TS, Eidelman EM, Litt AW, Pinto RS, Oldford F, Schwartzenberg T: Comparison of magnetic resonance angiography, conventional angiography, and duplex scanning. Stroke 1992; 23: 341-346.

17. Moneta GL, Edwards JM, Chitwood RW: Correlation of North American Symptomatic Carotid Endarterectomy Trial (NASCET) angiographic definition of $70 \%$ to $99 \%$ internal carotid artery stenosis with duplex scanning. J Vasc Surg 1993; 17: 152-159.

18. Cartier R, Cartier P, Fontaine A: Carotid endarterectomy without angiography. The reliability of doppler ultrasonography and duplex scanning in preoperative assessment. CJS 1993; 36: 411416.

19. Buchan A, Gates P, Pelz D, Barnett HJM: Intraluminal thrombus in the cerebral circulation. Stroke 1988; 19: 681-687.?

20. Eliasziw M, Streifler JY, Fox AJ, Hachinski VC, Ferguson GG, Barnett HJM: Significance of plaque ulceration in symptomatic patients with high-grade carotid stenosis. Stroke 1994; 25: 304308.

21. McCrory DC, Goldstein LB, Samsa GP, et al: Predicting complications of carotid endarterectomy. Stroke 1993; 24: 1285-1291. 